
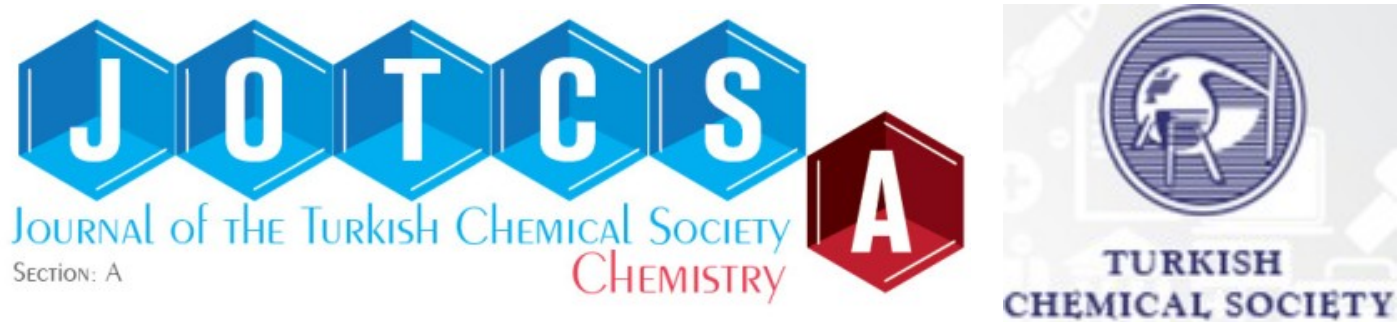

\title{
Sodium Borohydride and Essential Oils as Reducing Agents for the Chemically and Green Synthesis of Silver Nanoparticles: A Comparative Analysis
}

\author{
Pelin ERKOC \\ Faculty of Engineering and Natural Sciences, Bahcesehir University, Istanbul, Turkey.
}

\begin{abstract}
Silver nanoparticles (Ag-NPs) have gained immense attention among the variety of metallic nanoparticles during the past decades. In addition to the distinctive optical and electrical properties, Ag-NPs have been particularly employed for their biocidal features in industrial products, especially in biomedicine. Despite the wide range of studies and applications of Ag-NPs, yet serious limitations remained unsolved such as environmental toxicity caused by the reaction byproducts. In the present study, Ag-NPs synthesized by chemical synthesis as well by green synthesis method as a safer alternative considered as environmentally friendly, cost-effective and easy-to-handle. Sodium borohydride $\left(\mathrm{NaBH}_{4}\right)$ and the natural essential oils originating from rosemary (Rosmarinus officinalis) and grape seed (Vitis vinifera) were used as synthetic and bio-reducing agents, respectively. Ultraviolet-visible (UV-Vis) and X-ray Diffraction (XRD) spectroscopy analysis were performed, providing an insight into the composition of the Ag-NPs. Ultimately, the antimicrobial activity of the nanoparticles was tested on a gram-negative bacterium, Escherichia coli (E. coli). The results revealed that Ag-NPs can be synthesized by a sustainable alternative method with the usage of essential oils originating from rosemary and grape seed, and the resultant Ag-NPs exhibit enhanced the antimicrobial activity compared to the ones synthesized by the chemical reduction method.
\end{abstract}

Keywords: Silver nanoparticles, sodium borohydride, green synthesis, essential oils, antibacterial activity.

Submitted: May 14, 2020. Accepted: November 03, 2020.

Cite this: Erkoc P. Sodium Borohydride and Essential Oils as Reducing Agents for the Chemically and Green Synthesis of Silver Nanoparticles: A Comparative Analysis. JOTCSA. 2021;8(1):1-8.

DOI: https://doi.org/10.18596/jotcsa.737566.

*Corresponding author. E-mail: pelin.erkoc@eng.bau.edu.tr.

\section{INTRODUCTION}

Recent advances in nanotechnology have paved the way for the development of nanoparticles for a broad range of applications including health-care, food, and consumer products (1). Among metallic nanoparticles, silver nanoparticles (Ag-NPs) have been widely used because of their unique properties such as large specific surface area, catalytic activity, and antimicrobial activity (2). Furthermore, Ag-NPs have special optical features that cause localized surface plasmon resonance (LSPR) phenomenon to occur. When exposed to a particular wavelength, the electrons at the surface of the NPs undergo a collective oscillation. Owe to their strong interactions with light, they often used for biosensing applications (3).

Ag-NPs can be synthesized by "top-down" and "bottom-up" approaches, in which size of bulk materials reduced into fine particles, and selfassembly of atoms occurs to form a new nuclei that grow into a nanoparticle, respectively (2). In bottom-up approach, the most common method for the synthesis of Ag-NPs is chemical reduction using sodium borohydride $\left(\mathrm{NaBH}_{4}\right)$ as a reducing agent (4). However, biological methods are environmentally benign and more cost-effective over synthetic methods (5). This comparatively new method, known as green synthesis route, utilizes non-toxic substances derived from 
bioresource varying from plant extracts to microorganisms (6-9). The secondary metabolites found in plants, especially the terpenoid-rich essential oils, have a substantial role in Ag-NPs synthesis and attracted considerable attention in recent years (10). Essential oils can be extracted from plants such as rosemary, sage, thyme, and curcuma readily available worldwide as seasonings (6), and agricultural waste materials such as grape seeds (11). Moreover, these essential oils have been exhibited intrinsic anti-inflammatory, antioxidant, and antibacterial activities $(12,13)$. In this study, the aim was to conduct a comparative analysis on the synthesis routes of Ag-NPs. To achieve this goal, a study plan was developed including the synthesis of Ag-NPs by two different bottom-up approach, characterizations, and investigation of antibacterial activity on Escherichia coli ( $E$. coli). The present work focuses more on the green synthesis route of Ag-NPs with a dual combination of essential oils originating from rosemary and grape seed, using a Y-junction glass equipment.

\section{MATERIALS AND METHODS}

\section{Synthesis of Ag-NPs}

Silver nitrate $\left(\mathrm{AgNO}_{3}\right)$ of $99.9 \%$ purity (Sigma Aldrich) was the precursor for both synthetic routes. Deionized water $\left(\mathrm{dH}_{2} \mathrm{O}\right)$ was used in all experiments.

\section{Chemical synthesis of Ag-NPs}

Aqueous solutions of $0.35 \mathrm{M} \mathrm{AgNO}_{3}$ was prepared by dissolving in $50 \mathrm{~mL}$ of $\mathrm{dH}_{2} \mathrm{O}$. Then, an equal amount of $\mathrm{dH}_{2} \mathrm{O}$ in a separate flask was degassed by $\mathrm{N}_{2}$ gas for 30 minutes to provide an inert environment, and $1 \mathrm{M}$ of $\mathrm{NaBH}_{4}$ (\%96, Sigma Aldrich) solution was prepared.

A custom-made Y-junction glass equipment was used in the reaction to increase the yield $(14,15)$. $\mathrm{NaBH}_{4}$ and $\mathrm{AgNO}_{3}$ solutions were repelled through the center of flow tubes by $\mathrm{N}_{2}$ gas pressure, then a controlled molarity of chemical reacts when they encounter with each other. Reaction products at the neck were dropped into the collection beaker which contains the solvent (Scheme 1). The solvent used in reaction was added to the collection beaker and stirred with overhead stirrer (Thermomac) from start of the experiment to prevent aggregation. The product was then washed with acetone by centrifugation at 4100 rpm (Nuve NF400) for 10 minutes for 3 times and dried at room temperature under a laminar flow hood.

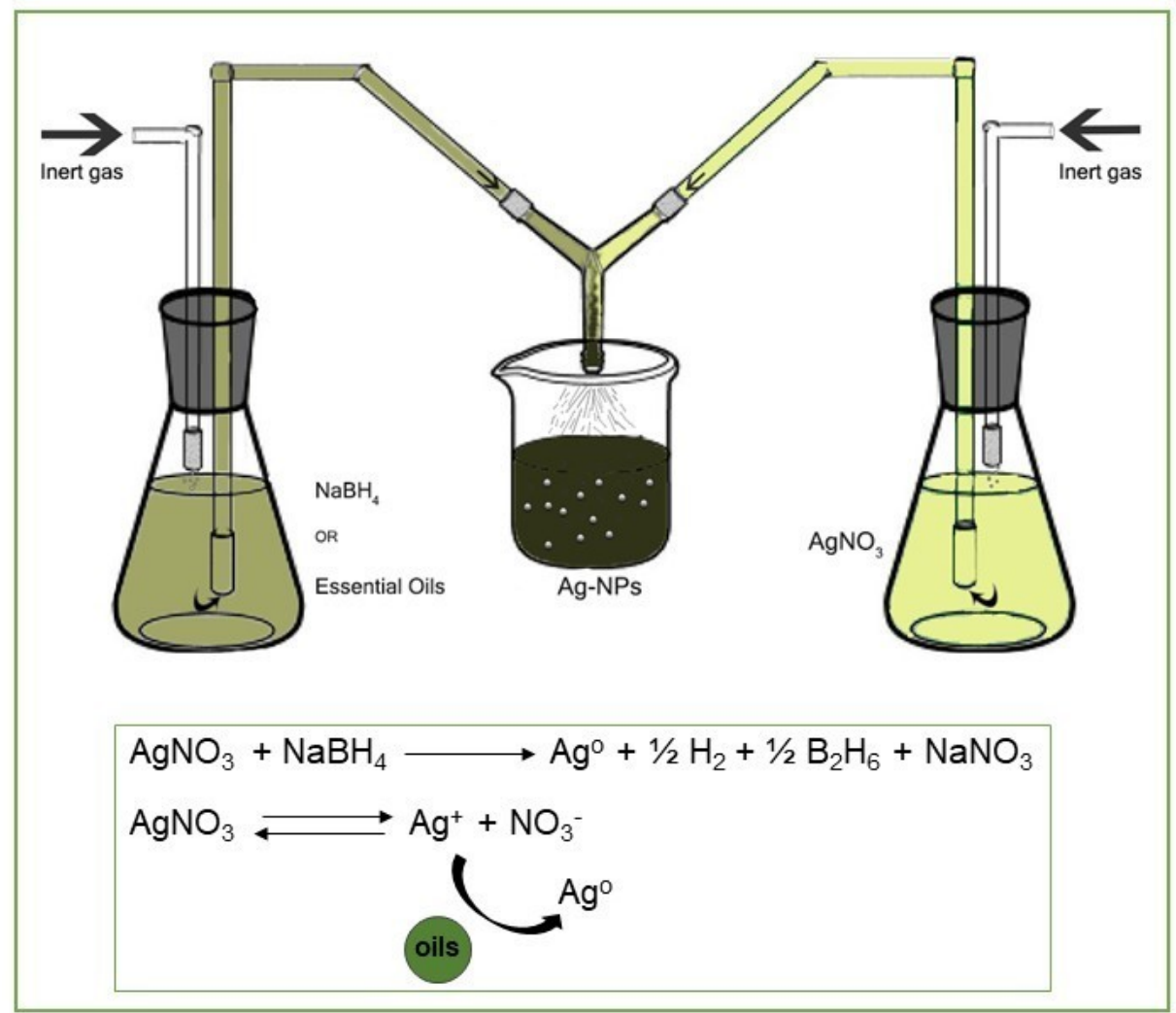

Scheme 1: Schematic representation of the synthesis of Ag-NP.

Green synthesis of Ag-NPs 
In the present study, essential oil reduction method is first time used with a $Y$-junction glass equipment to synthesize silver nanoparticles (AgNPs). Grape seed and rosemary essential oil were together used to prepare a solution that both reduces and stabilizes silver ions. Grape seed and rosemary essential oil mixture was prepared by diluting $1 \mathrm{~mL}$ of each oil together in $170 \mathrm{~mL}$ of acetone with 1 hour of constant stirring at room temperature. $\mathrm{AgNO}_{3}$ solution $\left(2.14 \times 10^{-4} \mathrm{M}, 50\right.$ $\mathrm{mL}$ ) was heated until its boiling point and $\mathrm{pH}$ is adjusted to 7 with $4 \mathrm{M} \mathrm{NaOH}$ solution. Then, as similar with the procedure used in chemical reduction route, equal volumes of essential oil and $\mathrm{AgNO}_{3}$ solutions were reacted in $\mathrm{Y}$-junction tube (Scheme 1).

The color change from transparent to yellowishbrown represents the Ag-NP formation. The product was then washed with acetone by centrifuging at $15,000 \mathrm{rpm}$ for 20 minutes for 3 times and dried at room temperature under a laminar flow hood.

\section{Characterizations}

UV-Vis absorbance measurement

UV-Vis absorption spectra of Ag-NPs in deionized water were measured in disposable polystyrene cuvettes (Isolab, Germany) by a T70 UV - Vis spectrophotometer (PG Instruments) with $0.5 \mathrm{~nm}$ spectral resolution.

\section{$X R D$ analysis}

$X$-ray Diffraction (XRD) spectroscopic analysis of the air-dried samples were performed by a Bruker D2 Phaser XRD.

\section{Antibacterial activity}

The in vitro antibacterial efficacy of the synthesized Ag-NPs was studied by the disk diffusion method using Luria-Bertani (LB) agar with determination of inhibition zones. First, an overnight culture of Stbl $3^{\mathrm{TM}} E$. coli strain (Thermo Fischer Scientific) was prepared from the isolated colonies selected from a 24-h grown LB agar plate (16), and optical density (OD) value was adjusted to 0.6 , which approximately corresponds to $1.5 \mathrm{x}$ $10^{8}$ colony-forming units (CFU) per milliliter of the bacteria suspension, by the spectrophotometric measurement at $600 \mathrm{~nm}$ (17). Afterwards, the antimicrobial susceptibility disks immersed in nanoparticle containing solutions ( $7.2 \mathrm{ng} / \mathrm{mL}$ in LB) were placed at on the agar dishes. Petri dishes were incubated at $37^{\circ} \mathrm{C}$ for 24 hours.

\section{RESULTS AND DISCUSSION}

There are numerous approaches for the synthesis of silver nanoparticles of various shapes and sizes (18). In the present study, the antibacterial activity of silver nanoparticles, which were synthesized via reduction of silver nitrate, using both chemical and green reducing agents, were evaluated. $\mathrm{NaBH}_{4}$ is utilized as a chemical reducing agent, whereas essential oils from two plant species, rosemary, and grape seed, were used as green reducing agents to combine their reducing capabilities. In contrast to current studies, here, a comparison-based methodology with a different experimental setup was carried out.

The methodology used in this work to obtain AgNPs is illustrated in Scheme 1 . The reaction apparatus, a Y-junction flow tube, used for the particles synthesis is a previously optimized technique for the synthesis of iron nanoparticles (15). In this system the apparatus contains two distillation flasks for the silver salt and reducing agent. The reduction reaction started as soon as the two solutions react in the neck of the flow tube whose size was kept as small as possible $(2 \mathrm{~cm})$ to prevent agglomeration.

The formation of Ag-NPs was first visually observed by the color change of the reaction mixtures. The transparent color of the silver nitrate solution changed to brownish-yellow during the reaction, indicating the growth of silver particles (19).

Moreover, UV-Vis spectra of the colloidal solutions obtained from the two routes of synthesis were shown in Figure 1. The LSPR absorption band, which is characteristic to silver nanoparticles, were observed for both samples around $400 \mathrm{~nm}$ (5, 20, 21). 
A

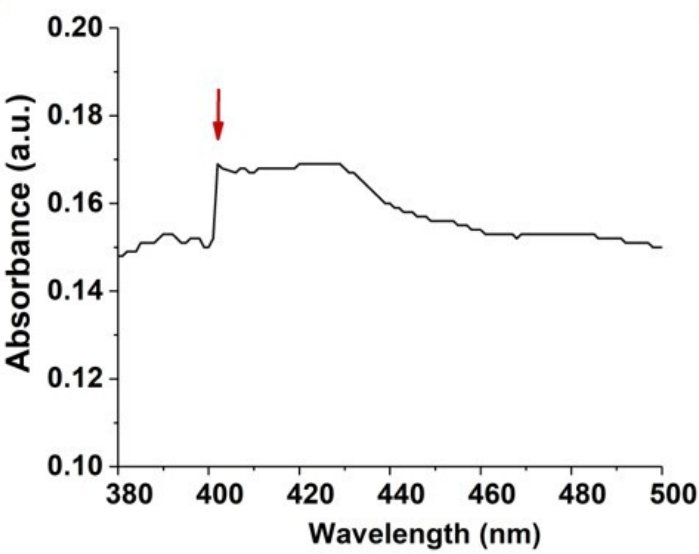

B

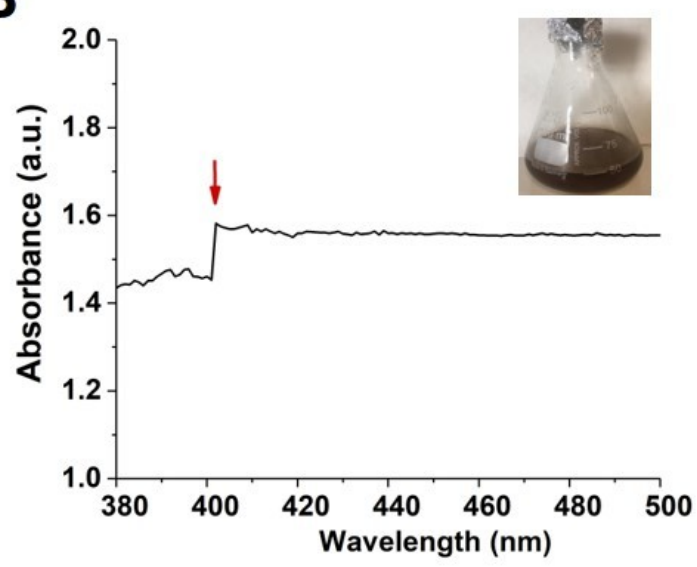

Figure 1. UV-Vis spectra. Silver nanoparticles synthesized by A) $\mathrm{NaBH}_{4}$ reduction and B) green synthesis.

Besides, UV-Vis spectral analysis shows that the values of absorbance corresponding to the $\lambda$ max for each $\mathrm{Ag}$ colloid were different. Higher absorbance value of Ag colloid synthesized by the chemical reduction method is taught to be resulted from the higher number of synthesized particles which highly depends on the differences in initial molar ratios of reactants used for these two methods.
The formation of the silver crystalline structures was evaluated by XRD spectroscopy. Figure 2 shows the XRD patterns of the formed Ag-NPs. The XRD peaks in the wide angle range of $2 \theta\left(30^{\circ}<2 \theta\right.$ $<80^{\circ}$ ) revealed that the peaks in $38.2^{\circ}, 46.2^{\circ}$, $64.1^{\circ}$ and $77.2^{\circ}$ can be attributed to the 111,200 , 220, and 311 crystalline structures of the face centered cubic (fcc) synthesized silver nanocrystal, respectively (17).

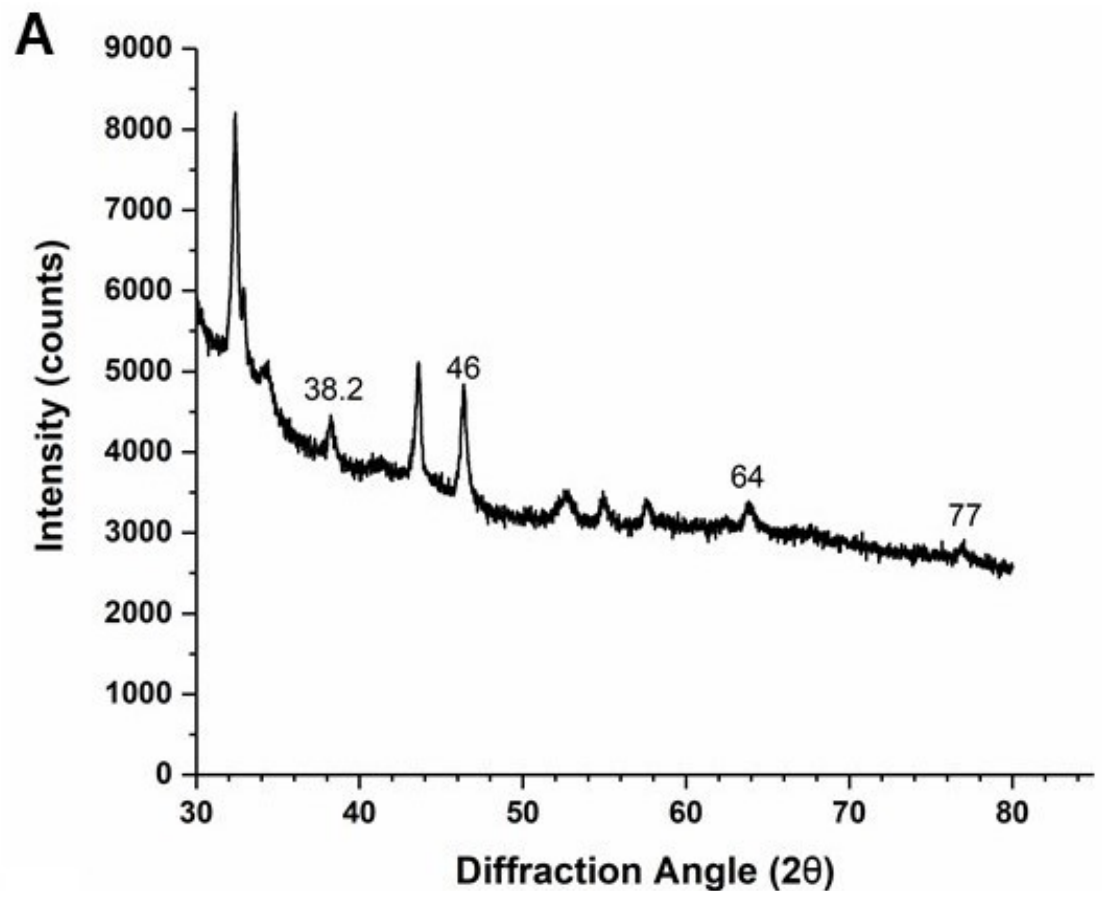




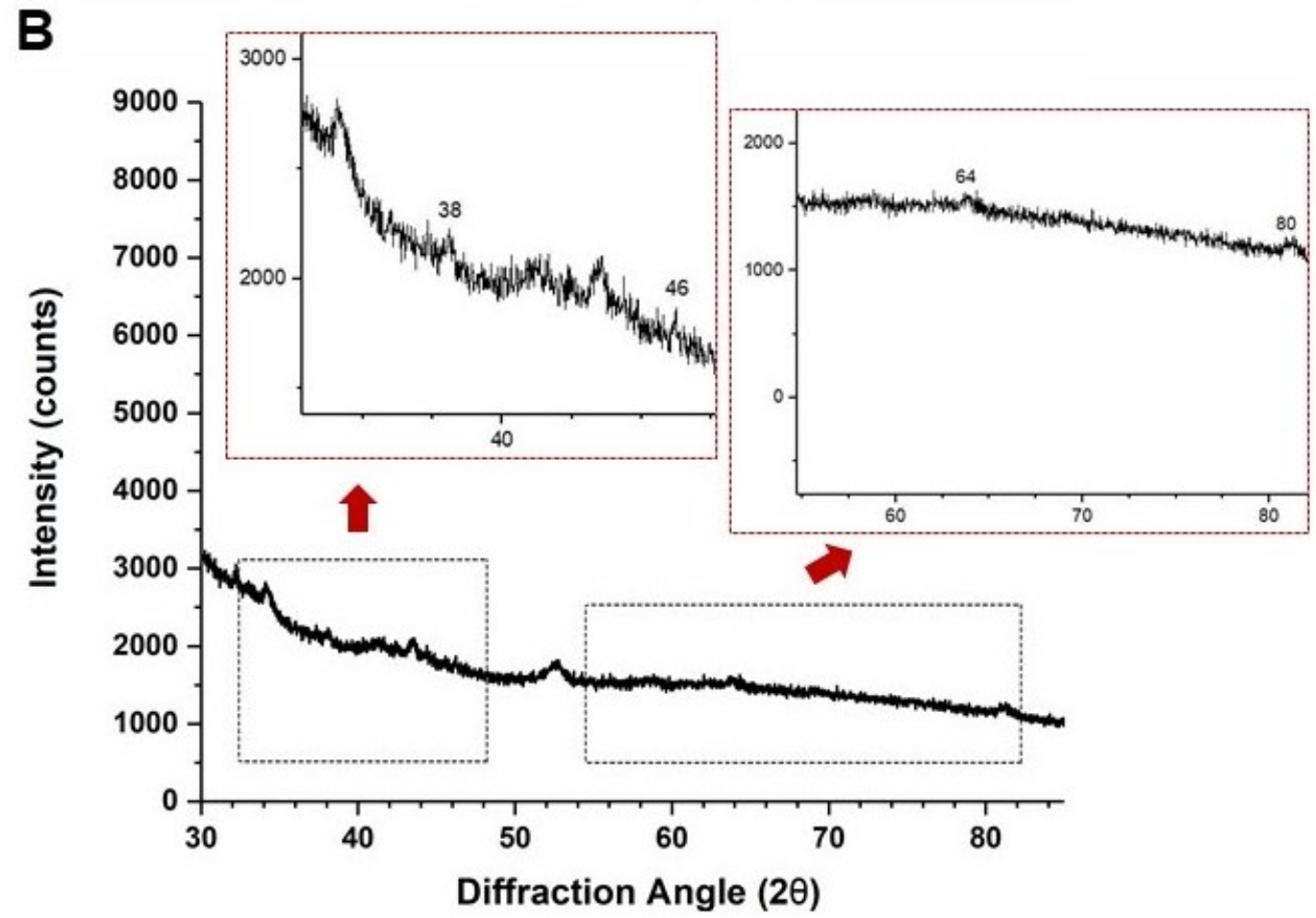

Figure 2. XRD patterns. Silver nanoparticles synthesized by $\mathbf{A}$ ) $\mathrm{NaBH}_{4}$ reduction and B) green synthesis.

Like the above discussed UV-Vis spectral analysis results, the corresponding XRD peaks were sharper and more visible for the particles synthesized by $\mathrm{NaBH}_{4}$ reduction. Hence, the average size of $\mathrm{Ag}-$ NPs synthesized by this method was calculated using Debye-Scherrer equation:

$$
D=\frac{K \times \lambda}{\beta \times \cos \theta}
$$

where $\mathrm{K}$ is the Scherrer constant with value from 0.9 (shape factor), $\lambda$ is the $X$-ray wavelength $(1.5418 \AA), \beta$ is the width of the XRD peak at half height and $\theta$ is the Bragg angle $(14,22)$. From the Scherrer equation, the average crystallite size of $\mathrm{Ag}-\mathrm{NPs}$ is found to be around $10-22 \mathrm{~nm}$.

As the final goal, the antibacterial activity of AgNPs was tested against a gram-negative bacterium, E. coli. Silver has already been known as an antibacterial agent since ancient times and it has been used in a variety of medical devices, such as catheters and in antimicrobial wound dressings (23). The antimicrobial activity of Ag, which lead to bacterial cell death, arises from the interaction of $\mathrm{Ag}^{+}$ions with thiol ( $\left.-\mathrm{SH}\right)$ groups of vital bacterial enzymes and proteins (3). In addition, free radicals of $\mathrm{Ag}$ are reported to react with the membrane lipids, causing damage to the membrane (23).
Disk diffusion or Kirby-Bauer susceptibility test is a routinely applied assay in clinical laboratories to detect the sensitivity or resistance of pathogenic bacteria to numerous antimicrobial compounds. In this assay, the presence or absence of microbial growth around the antimicrobial agent-soaked filter paper disk is an indirect technique to measure the ability of that agent to inhibit microbial growth on solid agar (24). Liquid medium assays are also preferred to determine the minimum inhibitory concentration (MIC) of an antimicrobial agent, which is the lowest concentration of the antimicrobial agent that inhibits growth (25). Utilization of disk diffusion on solid medium to determine zone of inhibition (zoi) remains as a widespread antimicrobial susceptibility testing tool, for being a standardized, easy, and low-cost method (25).

In this study, the antibacterial susceptibility was evaluated by the disk-diffusion assay (Figure 3 ). The results confirmed that $E$. coli exhibited sensitivity to Ag-NPs synthesized by the two techniques. Figure $3 \mathrm{C}$ shows that Ag-NPs synthesized by green synthesis method using essential oils originating from rosemary and grape seeds demonstrated increased antibacterial activity in a concentration dependent manner, whereas the difference between the diameters of growth inhibition zones were insignificant for NPs synthesized by chemical reduction using $\mathrm{NaBH}_{4}$. Previously, Raza et al. reported that the smallest- 
sized spherical Ag-NPs demonstrated a better antibacterial activity against E. coli (26). The difference in the inhibition efficiency of the Ag-NPs synthesized here might be due to the alterations in size and shape of NPs. Furthermore, in addition to the bio-reductant roles of essential oils used in this synthesis route, they also possess a broad spectrum of pharmacological impacts, such as antibacterial and antifungal activity (12, 13, 27), which can contribute to the higher antibacterial activity of the Ag-NPs synthesized by green chemistry.

\section{CONCLUSION}

This study represents a comparative analysis on the synthesis of silver nanoparticles using the chemical reduction and greener approach. In the present study, rosemary and grape seed essential oils were successfully used as green and sustainable reducing agents to synthesize silver nanoparticles. The antibacterial susceptibility of the silver nanoparticles was approved by the diskdiffusion on solid agar, which could be further tested by MIC assay in a quantitative way. Moreover, our preliminary conclusions are open to be extended by elaborative investigation of size distribution by SEM or TEM characterizations in a future study.

In summary, the results reinforced the idea that organic substances such as essential oils in plant extracts can be used for the synthesis of Ag-NPs. Finally, this study could provide an understanding of the green synthesis reactions, and it could provide a new avenue for nanoscience community and pharmaceutical industry to develop simple, safer, economical, and environmentally friendly methods for Ag-NP incorporated therapeutics.

\section{ACKNOWLEDGEMENTS}

The author would like to thank undergraduate students Merve Begum Bacinoglu, Sude Beskardes, Huseyin Berkcan İsilgan, and Assoc. Prof. Dr. Ozan Akdogan for their technical supports, Chemistry and Molecular Biology research facilities of Bahçeşehir University as well.
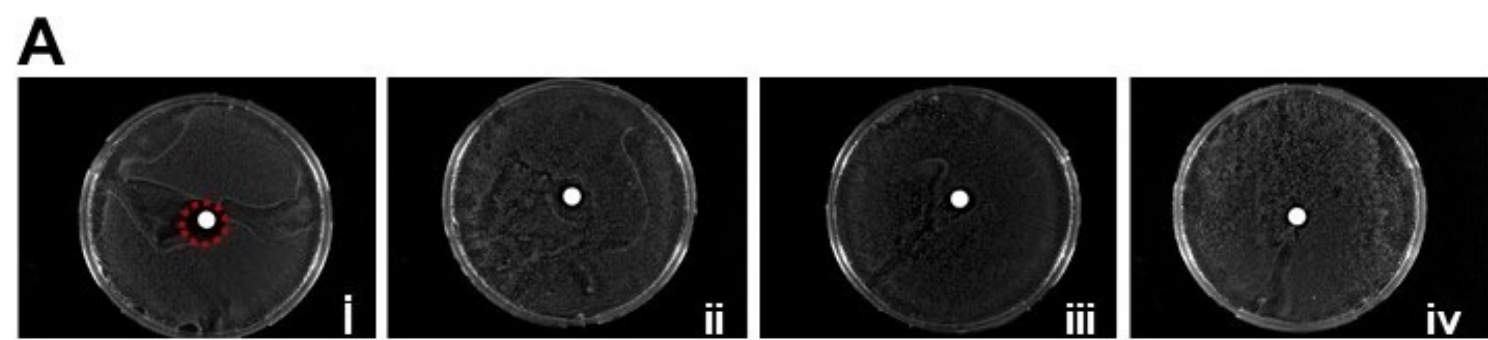

B
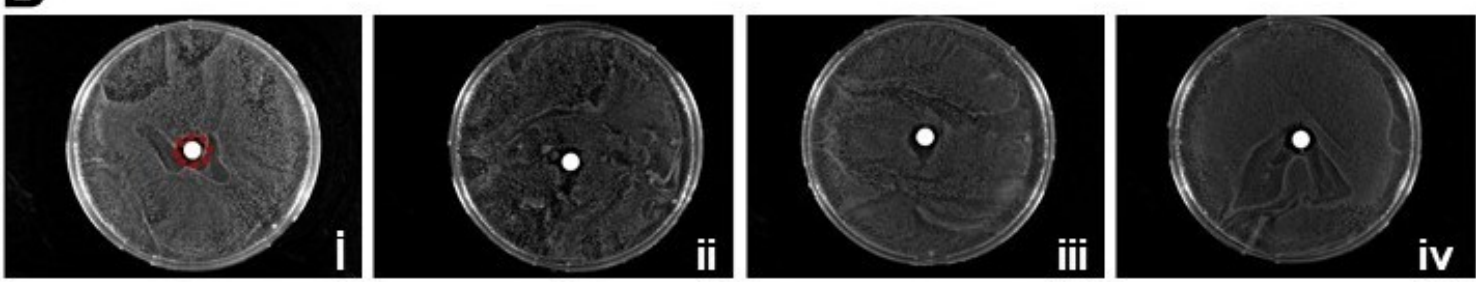


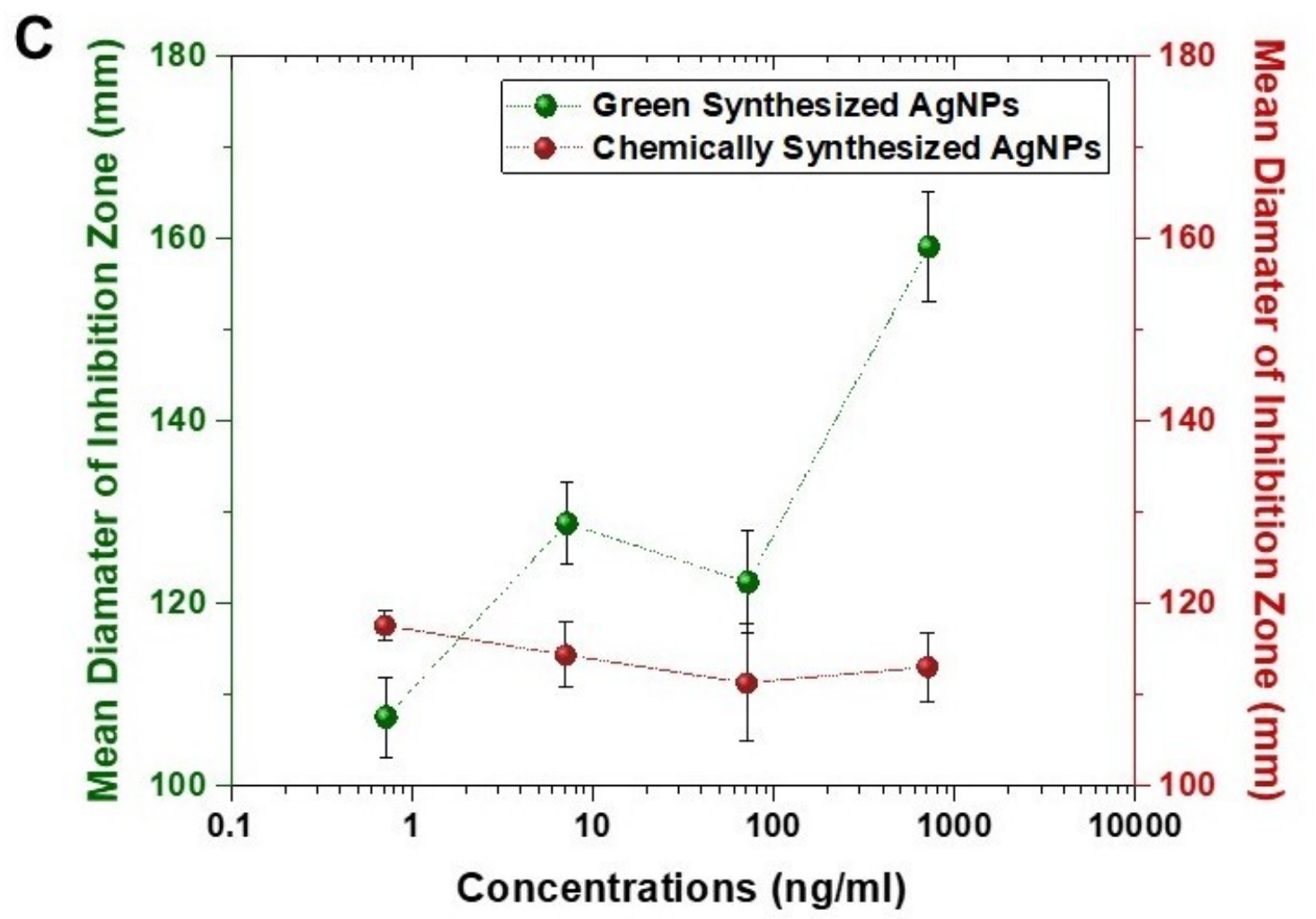

Figure 3. Disk diffusion assay. Antibacterial effect of silver nanoparticles synthesized by $\mathrm{A}$ ) $\mathrm{NaBH}_{4}$ reduction and B) green synthesis against $E$. coli. i-iv) Left to right increased concentrations of NPs are $720,72,7.2$ and $0.72 \mathrm{ng} / \mathrm{mL}$, respectively. C) Corresponding mean zone inhibition diameters. Diameters were manually measured by Image $\mathrm{J}$ software from 4 directions.

\section{REFERENCES}

1. Zhang XF, Liu ZG, Shen W, Gurunathan S. Silver Nanoparticles: Synthesis, Characterization, Properties, Applications, and Therapeutic Approaches. Int J Mol Sci. 2016;17(9):1534-68.

2. Rafique $M$, Sadaf I, Rafique MS, Tahir MB. A review on green synthesis of silver nanoparticles and their applications. Artificial Cells Nanomedicine Biotechnology. 2017;45(7):1272-91.

3. Loiseau A, Asila V, Boitel-Aullen G, Lam M, Salmain M, Boujday S. Silver-Based Plasmonic Nanoparticles for and Their Use in Biosensing. Biosensors (Basel). 2019;9(2):78-117.

4. Zhang Z, Shen W, Xue J, Liu Y, Liu Y, Yan $P$, et al. Recent advances in synthetic methods and applications of silver nanostructures. Nanoscale Research Letters. 2018;13(1):54-72.

5. Vilas V, Philip D, Mathew J. Essential oil mediated synthesis of silver nanocrystals for environmental, anti-microbial and antioxidant applications. Material Science and Engineering: C. 2016;61:429-36.
6. González-Rivera J, Duce C, Ierardi V, Longo I, Spepi A, Tiné MR, et al. Fast and Ecofriendly Microwave-Assisted Synthesis of Silver Nanoparticles using Rosemary Essential Oil as Renewable Reducing Agent. ChemistrySelect. 2017;2(6):2131-8.

7. Korkmaz N. Bioreduction; The Biological Activity, Characterization and Synthesis of Silver Nanoparticles. Turkish Journal of Chemistry. 2020.

8. Sahin M, Gubbuk IH. Green Synthesis of Antioxidant Silver and Platinum Nanoparticles Using Ginger and Turmeric Extracts and Investigation of Their Catalytic Activity. Journal of the Turkish Chemical Society A: Chemistry. 2019;6(3):403-10.

9. Shelar A, Sangshetti J, Chakraborti S, Singh AV, Patil R, Gosavi S. Helminthicidal and Larvicidal Potentials of Biogenic Silver Nanoparticles Synthesized from Medicinal Plant Momordica charantia. Med Chem. 2019;15(7):7819.

10. Mashwani ZU, Khan MA, Khan T, Nadhman A. Applications of plant terpenoids in the synthesis of colloidal silver nanoparticles. Advances in Colloid and Interface Science. 2016;234:132-41. 
11. Ping $Y$, Zhang $\mathrm{J}$, Xing $\mathrm{T}$, Chen $\mathrm{G}$, Tao $\mathrm{R}$, Choo K-H. Green synthesis of silver nanoparticles using grape seed extract and their application for reductive catalysis of Direct Orange 26 . Journal of Industrial and Engineering Chemistry. 2018;58:749.

12. Dzimitrowicz $A$, Berent $S$, Motyka $A$, Jamroz $\mathrm{P}$, Kurcbach $\mathrm{K}$, Sledz W, et al. Comparison of the characteristics of gold nanoparticles synthesized using aqueous plant extracts and natural plant essential oils of Eucalyptus globulus and Rosmarinus officinalis. Arabian Journal of Chemistry. 2019;12(8):4795-805.

13. Oroojalian $F$, Orafaee $H$, Azizi $M$. Synergistic antibaterial activity of medicinal plants essential oils with biogenic silver nanoparticles. Nanomedicine Journal. 2017;4(4):237-44.

14. Erkoc $\mathrm{P}$, Odeh $\mathrm{YN}$, Alrifai $\mathrm{N}$, Zirhli $\mathrm{O}$, Gunduz Akdogan N, Yildiz B, et al. Photocurable pentaerythritol triacrylate/lithium phenyl-2,4,6trimethylbenzoylphosphinate-based ink for extrusion-based 3D printing of magneto-responsive materials. Journal of Applied Polymer Science. 2020:49043.

15. Khurshid H. Synthesis and Characterization of Iron Based Nanopartiles for Novel Applications [PhD]. UMI Dissertations Publishing: University of Delaware; 2011.

16. Elbing KL, Brent R. Recipes and Tools for Culture of Escherichia coli. Current Protocols in Molecular Biology. 2019;125(1):e83-97.

17. Shameli $K$, Ahmad MB, Jazayeri SD, Shabanzadeh $P$, Sangpour $P$, Jahangirian $H$, et al. Investigation of antibacterial properties silver nanoparticles prepared via green method. Chemistry Central Journal. 2012;6(1):73-83.

18. Mousavi-Khattat M, Keyhanfar M, Razmjou A. A comparative study of stability, antioxidant, DNA cleavage and antibacterial activities of green and chemically synthesized silver nanoparticles. Artificial Cells, Nanomedicine, and Biotechnology. 2018;46(sup3):S1022-S31.

19. Ga'al H, Fouad H, Mao G, Tian J, Jianchu M. Larvicidal and pupicidal evaluation of silver nanoparticles synthesized using Aquilaria sinensis and Pogostemon cablin essential oils against dengue and zika viruses vector Aedes albopictus mosquito and its histopathological analysis. Artificial Cells Nanomedicine Biotechnology. 2018;46(6):1171-9.

20. Junejo Y, Baykal A. Ultrarapid catalytic reduction of some dyes by reusable novel erythromycin-derived silver nanoparticles. Turkish Journal of Chemistry. 2014;38:765-74.

21. Singh AV, Bandgar BM, Kasture M, Prasad BLV, Sastry M. Synthesis of gold, silver and their alloy nanoparticles using bovine serum albumin as foaming and stabilizing agent. J Mater Chem. 2005;15(48):5115-21.

22. Kalishwaralal $\mathrm{K}$, Deepak $\mathrm{V}$, Ramkumarpandian S, Nellaiah H, Sangiliyandi G. Extracellular biosynthesis of silver nanoparticles by the culture supernatant of Bacillus licheniformis. Materials Letters. 2008;62(29):4411-3.

23. Granbohm H, Larismaa J, Ali S, Johansson LS, Hannula SP. Control of the Size of Silver Nanoparticles and Release of Silver in Heat Treated $\mathrm{SiO}(2)-\mathrm{Ag}$ Composite Powders. Materials 2018;11(1):80-97.

24. Hombach $M$, Zbinden $R$, Böttger EC. Standardisation of disk diffusion results for antibiotic susceptibility testing using the sirscan automated zone reader. BMC Microbiology. 2013;13(1):225.

25. Flanagan JN, Steck TR. The Relationship Between Agar Thickness and Antimicrobial Susceptibility Testing. Indian J Microbiol. 2017;57(4):503-6.

26. Raza MA, Kanwal Z, Rauf A, Sabri AN, Riaz $S$, Naseem S. Size- and Shape-Dependent Antibacterial Studies of Silver Nanoparticles Synthesized by Wet Chemical Routes. Nanomaterials-Basel. 2016;6(4):74-89.

27. Shrestha B, Theerathavaj MLS, Thaweboon $S$, Thaweboon B. In vitro antimicrobial effects of grape seed extract on peri-implantitis microflora in craniofacial implants. Asian Pac J Trop Biomed. 2012;2(10):822-5. 